\title{
APRENDIZAJE PROFESIONAL DOCENTE A TRAVÉS DE LA AUTOFORMACIÓN: UN ANÁLISIS CUALITATIVO DE LAS ECOLOGÍAS DE APRENDIZAJE
}

\author{
Iris Estévez ${ }^{1}$, Alba Souto-Seijo², Olalla Sande ${ }^{3}$ y Mercedes González-Sanmamed ${ }^{4}$ \\ Universidade da Coruña, España, ${ }^{1}$ iris.estevezb@udc.es; ${ }^{2}$ a.souto1@udc.es; ${ }^{3}$ o.sande@outlook.es; ${ }^{4}$ mercedes@udc.es
}

\begin{abstract}
Resumen. En la era de las tecnologías digitales, surgen nuevos, amplificados e híbridos contextos que proporcionan a los docentes múltiples formas de involucrarse y aprovechar las oportunidades de aprendizaje y desarrollo profesional. Una de las modalidades de formación más valoradas y utilizadas por los docentes es la autoformación, que se ha visto facilitada y enriquecida con la llegada de las herramientas digitales e Internet. El objetivo de este estudio es analizar y comprender cómo los docentes configuran y utilizan sus propias Ecologías de Aprendizaje para desarrollarse profesionalmente y, especificamente, mediante la modalidad de autoformación. Con este fin, se llevó a cabo un estudio de carácter cualitativo dentro de la tradición de investigación de Estudio de Caso. Nuestro caso se compone de cuatro docentes de Educación Infantil en activo. Para la recogida de información se realizaron dos entrevistas semiestructuradas con cada docente. Los resultados se estructuran en función de las subcategorías emanadas en torno al elemento de autoformación: recursos y mecanismos. En este apartado se describen los recursos y los mecanismos de autoformación más frecuentemente utilizados y más valorados en nuestro caso de estudio. Asimismo, se incide en la necesidad de una reformulación de los escenarios de formación.
\end{abstract}

Palabras clave: Desarrollo Profesional Docente; Ecologías de Aprendizaje; Autoformación; Estudio de Caso; Educación Infantil.

\section{PROFESSIONAL TEACHER LEARNING THROUGH SELF-DIRECTED TRAINING: A QUALITATIVE ANALYSIS OF LEARNING ECOLOGIES}

\begin{abstract}
In the era of digital technologies, new, amplified and hybrid contexts emerge. They provide teachers with multiple ways to get involved and take advantage of learning and professional development opportunities. One of the training methods most valued by teachers is self-directedtraining, which has been enriched by the legacy of digital tools and Internet. This study aims at analyze how teachers configure and use their own Learning Ecologies to develop professionally and, specifically, through the self-directed-training modality. Thus, we carried out a qualitative research, within the Case Study research tradition. Our case consists of four active Early Childhood Education teachers. For data collection, two semi-structured interviews were conducted with each teacher. We structured the results obtained according to subcategories emanating around the self-directedtraining element: resources and mechanisms. This section describes the resources and self-training mechanisms most frequently used and most valued in our case study. In addition, we also emphasize the need for a reformulation of training scenarios.
\end{abstract}

Keywords: Teacher Professional Development; Learning Ecologies; Self-directed Training; Case Study; Early Childhood Education.

\section{INTRODUCCIÓN}

En nuestra sociedad actual existe una necesidad de formación y de actualización continua que se incrementa en el caso de aquellas personas dedicadas profesionalmente a la educación (González-Sanmamed, Estévez, Souto-Seijo, \& Muñoz-Carril, 2020). Los docentes no sólo tienen la necesidad de aprender, sino también la responsabilidad de enseñar en un 
entorno en constante cambio. Así, en la era de las tecnologías sociales y ubicuas, estos nuevos, amplificados e híbridos contextos proporcionan a los individuos múltiples formas de involucrarse y aprovechar las oportunidades de aprendizaje y desarrollo profesional. De este modo, a medida que las Tecnologías de la Información y Comunicación (TIC) se han ido desarrollando, han ido emergiendo nuevas posibilidades de aprendizaje que exceden los límites de la formación formal o institucional (Sangrà, Estévez, Iglesias, \& Souto-Seijo, 2019). Como pusieron de manifiesto González-Sanmamed, Sangrá, Souto-Seijo y Estévez (2018), uno de los catalizadores que impulsaron la metamorfosis de los procesos de aprendizaje fue la incursión de las tecnologías digitales e Internet, puesto que han supuesto la ruptura de las barreras espacio-temporales en los contextos formativos. Este hecho nos hace dirigir la mirada hacia lo que Cope y Kalantiz (2010) denominaron aprendizaje ubicuo, que se vincula con la idea de "aprender en cualquier momento y en cualquier lugar".

Derivado de lo anterior y siguiendo con la propuesta de González-Sanmamed et al. (2018), se determina como otro de los catalizadores del cambio en los sistemas de aprendizaje de los individuos, la generación de nuevos y diversos contextos para desarrollarse y aprender. En esta línea Castañeda y Adell (2013) hacen una interesante propuesta para definir los diferentes escenarios de aprendizaje de los docentes, tomando como criterio de análisis dos ejes: enseñanza estructurada / sin estructurar y aprendizaje planificado/ sin planificar. Así, se da lugar a cuatro tipologías clave: formal, no formal, informal y autodidacta (Castañeda, \& Adell, 2013, p. 104). En este estudio vamos a focalizar nuestra mirada hacia este último escenario de aprendizaje. La autoformación y el aprendizaje autodidacta se pueden dar en cualquier lugar, y no se cierne a las barreras de las instituciones formales de formación. Sin embargo, a diferencia del aprendizaje informal, sí obedece a una planificación de objetivos de aprendizaje, así como a la consecución de metas establecidas por el propio aprendiz. En el aprendizaje autodidacta sí hay una total consciencia y volición de aprender, a diferencia del aprendizaje informal (Ibid, 2013). Para Guglielmino (2008) este tipo de aprendizajes dirigidos por uno mismo, representan la respuesta más natural cuando nos enfrentamos a dificultades, retos o nuevos fenómenos, especialmente en la actualidad, donde el acceso a la información y la comunicación es inmediato, y se exige una respuesta de adaptación rápida por parte de los individuos (Marcelo, 2010).

En este contexto, dónde existen tantas y tan diversas posibilidades para que los docentes sigan formándose a lo largo de su trayectoria profesional y personal, surge la metáfora ecológica como perspectiva de análisis del aprendizaje de los individuos. Las Ecologías de 
Aprendizaje definidas como "los procesos creados en un contexto particular para un propósito particular que brinda oportunidades, relaciones y recursos para el aprendizaje, el desarrollo y los logros" (Jackson, 2013, p.14), facilitan un enfoque capaz de integrar todas las oportunidades de formación, teniendo en cuenta el rico y diverso conjunto de posibilidades de aprendizaje disponibles en la era digital. De acuerdo con Maina \& García (2016), y Sangrá, González-Sanmamed y Guitert (2013), ser conscientes de cómo se configuran nuestras ecologías puede ser una estrategia muy útil que nos ayude a desarrollarnos tanto personal como profesionalmente.

El objetivo principal de este estudio es analizar y comprender cómo los docentes configuran y utilizan sus propias Ecologías de Aprendizaje para desarrollarse profesionalmente. Concretamente, los objetivos específicos giran en torno al análisis de los recursos y mecanismos que emplean para su autoformación desde una perspectiva ecológica.

\section{METODOLOGÍA}

La investigación se enmarca en una perspectiva de investigación cualitativa a través de entrevista. Concretamente la tradición de investigación seleccionada para el desarrollo de este trabajo es el Estudio de Caso (Stake, 2005,2010). Esta es una tradición de investigación que permite el análisis en profundidad de realidades sociales particulares (Jorrín-Abellán, 2016) y contribuye al estudio de sistemas bien delimitados en acción (MacDonald \& Walker, 1975).

\subsection{Participantes}

Los participantes de nuestro estudio han sido cuatro docentes de Educación Infantil en activo (Zeltia, Nicolás, Sara y Ana) pertenecientes a diferentes centros públicos de la Provincia de A Coruña (España). Tres de los cuatro profesores desempeñaban su tarea docente en el segundo ciclo de Educación Infantil y uno de ellos en el primer ciclo y poseen edades comprendidas entre 34 y 48 años. Tomando en consideración los principios éticos en torno a la protección de la privacidad y el anonimato de los participantes, hemos ocultado los nombres reales de los entrevistados, salvaguardando así su identidad (Creswell \& Poth, 2018). Se ha utilizado un muestreo de casos homogéneos (Patton, 2002) ya que se pretende describir un subgrupo de personas que comparten determinadas experiencias comunes en torno al núcleo de la investigación. Así pues, cabe mencionar algunos de los criterios de elegibilidad de los participantes, los cuales se muestran en la Figura 1. 
Participación en formación no reglada reglada

-Persona activa en la búsqueda de formación (cursos del CFR, de la universidad, online...). No sólo charlas sino también grupos de trabajo, seminarios, PFPP...

-Interacción con el entorno más próximo (compañeros/as, amistades...) con la finalidad de ampliar la formación.

-Perfil proactivo en su proceso de actualización y en el de los demás (p.e. ponentes)

\section{Competencias básicas}

-Empleo de metodologías innovadoras en el aula.

-Integrar en la programación de aula las competencias clave determinadas en la actual Ley Orgánica de educación (LOMCE)

\section{TIC}

-Elaboración de actividades propias a través de las TIC.

-Integración en la programación del uso de las TIC

-Alto nivel de competencia digital

\section{Impacto de la globalización}

- Colaboración en diferentes programas europeos o viajes con el fin de ampliar la formación (visitar escuelas de Finlandia, Reggio Emilia...).

-Empleo de eTwinning en el aula.

- Hacer partícipe al alumnado en programas europeos (p.e. Comenius).

Figura 1. Criterios de selección de los participantes de la investigación.

\subsection{Estrategias de Recogida de Información}

Se seleccionó la entrevista como instrumento de recogida de información por su pertinencia dado el fenómeno a estudiar. Entre los diferentes tipos de entrevista, nos decantamos por una entrevista semiestructurada, ya que, como explica Mayorga-Fernández (2004), se va adecuando a las respuestas que ofrece el entrevistado con el fin de obtener la mayor información posible sobre las cuestiones planteadas. Munarriz (1992) la define como una conversación cara a cara que mantienen entrevistador y entrevistado. El investigador diseña un guion de entrevista con una batería de cuestiones en base al conocimiento previo sobre el tópico, pero no es preciso seguir un orden rígido y las preguntas planificadas deben adaptarse a las respuestas de los entrevistados durante el desarrollo de la entrevista. Siguiendo estas directrices, se elaboraron dos guiones de entrevistas para abordar diferentes temáticas. En primer lugar, la entrevista de carácter biográfico con el objetivo de contextualizar la trayectoria 
personal y profesional del docente y recoger datos que nos ayuden a comprender su historia de vida y sus reflexiones sobre su desarrollo personal. En segundo lugar, la entrevista sobre las Ecologías de Aprendizaje, con el fin de indagar acerca de sus mecanismos para seguir formándose -formación permanente y actualización-, el empleo de las TIC para su formación, y qué ambientes o entornos frecuentan para autoformarse.

\subsection{Procedimiento}

En primer término, se estableció el contacto con los cuatro docentes seleccionados, invitándoles a formar parte de la investigación e indicándoles los objetivos de la misma. Además, fueron informados de los principios éticos que guiarían el desarrollo de la investigación, por lo que su identidad y los datos que se recogieran serían estrictamente confidenciales y solo se utilizarían con fines de investigación. Una vez obtuvimos la aceptación y confirmación de participación de los cuatro profesores, dispusimos una fecha y una ubicación para el desarrollo de las entrevistas. Las dos entrevistas se realizaron en una única sesión con cada docente. Todas las entrevistas fueron grabadas en audio, bajo el consentimiento de los participantes. Posteriormente, los datos recogidos fueron transcritos para proceder a su análisis.

\subsection{Análisis de Datos}

El análisis de datos en las corrientes cualitativas se basa en un proceso de interpretación, proporción de significados y categorización de la información recogida. Así pues, todo el proceso de análisis fue apoyado por el software Atlas.ti (Versión 7.3), y realizado por dos investigadoras. Asimismo, una de las peculiaridades de la investigación cualitativa es que se suele acumular gran cantidad de información a analizar. Es por ello que Miles, Huberman y Saldaña (2014) presentan un modelo de análisis que facilita el abordaje del conjunto de los datos de naturaleza textual. Este modelo presenta tres pasos recurrentes que constituyen el proceso analítico básico, común a la mayoría de los estudios en los que se trabaja con datos cualitativos. En primer lugar, la reducción de datos; en segundo lugar, la representación de los datos en formato síntesis, para finalmente dar paso a la elaboración de conclusiones. Así pues, el objetivo del análisis de las entrevistas era conocer en profundidad todos los elementos que conforman las Ecologías de Aprendizaje de los docentes entrevistados en su proceso de desarrollo profesional, centrándonos especialmente en aquellos componentes vinculados a la autoformación. 
Este procedimiento obedece a la estrategia de análisis de contenido. Nuestra aproximación parte de la creación de un sistema de categorías de análisis basado en la teoría acerca de las Ecologías de Aprendizaje y de los procesos de autoformación del profesorado. A partir de ahí se sucedió un proceso de análisis inductivo-deductivo en base al sistema de categorías creado previamente. Así, se fueron seleccionado porciones textuales e identificándolas con diferentes códigos. El sistema de categorías fue sufriendo cambios a medida que se codificaba y se releía el material. A término del proceso de codificación, emanó el siguiente esquema sistema de códigos (Figura 2) que vinculaba todos los fragmentos de los casos entrevistados sobre los tópicos de estudio: desarrollo profesional (DP) y Ecologías de Aprendizaje (EdA).

Teniendo en cuenta el objetivo de esta investigación, nos hemos centrado en la interpretación de los datos obtenidos en torno a la categoría de autoformación. Dónde se hace referencia a qué recursos y mecanismos emplean los docentes para desarrollarse profesionalmente $y$, por tanto, qué elementos autoformativos constituyen sus Ecologías de Aprendizaje. Destacar el uso de estrategias de triangulación de los datos observados y entre investigadores (Denzin, 2015) para la elaboración de conclusiones, lo que da soporte al rigor y credibilidad de la investigación

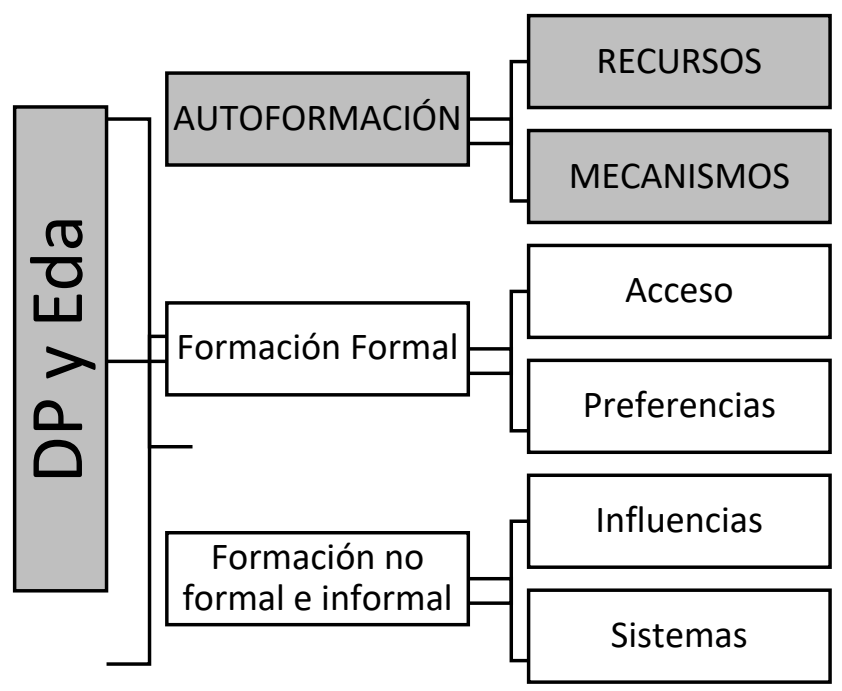

Figura 2. Sistema de Categorías emanado del análisis de datos. 


\section{RESULTADOS}

Los resultados se estructuran en función de las dos subdimensiones establecidas en el sistema de categorías. Por un lado, hablaremos de los recursos que emplean los docentes y, por otro lado, los mecanismos o las estrategias que ponen en marcha con fines de autoformación.

Dentro de la categoría recursos, destacan las herramientas digitales e Internet, puesto que tres de nuestros cuatro profesores determinan las tecnologías como los instrumentos imprescindibles en su autoformación. Así, nos valemos de las palabras de Zeltia para ilustrar esta cuestión: "siempre que tengo alguna temática en la cabeza, busco en algún blog de referencia o aleatoriamente en Internet, y siempre surgen" (E2- Z, 6)". Nicolás, por su parte, afirma lo siguiente "investigar en blogs, buscar actividades, recursos... el ordenador para mí es una herramienta clave. O el móvil que te permite en un momento concreto sacar una foto, buscar una información rápida..." (E2- N, 6); "sigo blogs y webs de gente que me interesa como docentes o psicólogos" (E2- N, 6); y Ana comenta "en las cosas que preparas para el aula, estás buscando información constantemente. Igual empiezas mirando inteligencias múltiples, encuentras un archivo, te lo lees, acabas viendo inteligencia emocional y terminas buscando más sobre eso..." (E2- A, 1). Otra de las cuestiones a resaltar viene de la mano de Sara, que entiende los viajes a otros centros, dentro del contexto nacional e internacional, como una de las fuentes de aprendizaje más valiosas, y así lo retratan sus palabras "es muy enriquecedor cuando visitas y te dan la oportunidad de formar parte de otra manera de ver... Es súper positivo lo de estar cambiando porque si no al final es como tu gueto y no sabes qué hay fuera. Compartir experiencias, conocer gente distinta... Es muy positivo a todos los niveles: para el aprendizaje y para la vida" (E2- S, 3).

Por otro lado, en cuanto a los resultados obtenidos sobre los mecanismos que los docentes ponen en marcha para autoformarse, uno elemento que destaca como estrategia de autoformación es la realización de registros o diarios de aula. Tres de los cuatro docentes realizan esta tarea diaria o semanalmente. Por ejemplo, Nicolás comenta lo siguiente: "Hacemos reseña de lo que hacemos de trabajo y de aspectos significativos que han pasado con nuestros alumnos" (E2- N, 7). Por su parte, Sara añade "intentamos registrar absolutamente todo lo que dicen o hacen los niños" (E2- S, 8). Otro de los mecanismos que los docentes señalan y califican como más eficaz y usual en su práctica es la reflexión, tanto individual como grupal. Todos coinciden en la reflexión individual como la estrategia de 
autoevaluación más eficaz y las palabras de Nicolás representan esta idea "ves que tienes algunas lagunas o que necesitas saber algo más, para mí ese es el motor de mi profesión y la motivación para formarme" (E2- N, 5). Por su parte, Zeltia subraya la valía de la reflexión personal "hago una evaluación global, no de forma escrita pero si pensando y reflexionando en qué puedes mejorar o algo que quieres profundizar porque fue bien, algo que quedó flojo o no enganchó bien..." (E2-Z, 5). Sara enfatiza la funcionalidad de la reflexión grupal, posterior a la individual "en todas esas reflexiones y autorreflexiones, de ahí es de dónde vas aprendiendo y vas diciendo -mira chicas esto no lo vamos a seguir haciendo porque no funciona, hay que cambiarlo- y buscamos propuestas para cambiarlo (E2- S, 8).

\section{CONCLUSIONES}

A través de este Estudio de Caso se ha observado cómo el uso de las tecnologías de la información y la comunicación fortalecen los procesos formativos y posibilitan una mejora de las competencias profesionales de los docentes de Educación Infantil. Así, esta investigación ha permitido identificar las diferentes oportunidades de actualización que existen dentro de la modalidad de autoformación, yendo más allá de las ofertas formativas de carácter formal o no formal.

En primer término, se concluye que los docentes que componen nuestro caso de estudio son profesionales muy implicados en su formación. En segundo término, centrándonos específicamente en las subdimensiones emergidas en torno al elemento autoformativo, se evidencia cómo los recursos que los docentes utilizan en mayor medida para autoformarse son las tecnologías digitales e Internet. En esta línea, destacan los blogs educativos como fuentes de información más recurridas por los docentes de nuestro estudio. Este hallazgo está en línea con lo aportado por González-Sanmamed, Souto-Seijo, et al. (2019), que afirman que la consulta de blogs es una de las actividades más realizadas por los docentes de Educación Infantil para mantenerse actualizados. Cabe destacar que estos espacios web tienen un gran peso en el ámbito educativo (Grané, 2009) y permiten al profesorado interactuar con otros profesionales con intereses similares e intercambiar conocimientos, lo que contribuye a mejorar su desarrollo profesional (Huei-Tse Hou, Kuo-En Chang, \& Yao-Ting Sung, 2009). Por otro lado, también es necesario subrayar que las estancias en otros centros internacionales y nacionales son entendidas como un recurso muy valioso de cara a la mejora y actualización profesional, aunque no se realice de forma habitual por su elevado coste, en términos de tiempo y esfuerzo. 
En cuanto a los mecanismos que los docentes de nuestro caso ponen en marcha para autoformarse, se muestra relevante la generación de diarios de clase, como estrategia de recogida de información, que da lugar, posteriormente, al inicio de procesos de reflexión sobre la información recogida. No en todos los casos se produce esa recogida de información sobre la propia práctica de forma sistematizada o altamente estructurada, pero sí que todos los docentes de nuestra investigación realizan ejercicios de reflexión sobre la acción. Como enuncia Marcelo (2010) es el análisis de la experiencia lo que posibilita cuestionar y formular interrogantes sobre las propias ideas y prácticas, de cara a incrementar la consciencia sobre las propias carencias en el conocimiento profesional. Siendo esta una estrategia altamente eficaz para mejorar el desempeño docente.

Por ello, se considera importante que las diferentes instituciones educativas tengan en consideración el valor atribuido a las experiencias de reflexión personal y grupal, ya que podría constituir un punto de partida para la generación de espacios donde este tipo de estrategias de aprendizaje se pudieran desarrollar con éxito. Además, estos resultados exhortan hacia un replanteamiento de las modalidades de formación que son promovidas, ya que, como hemos podido observar el profesorado reconoce y valora los escenarios autodidactas para la asunción de algunas competencias profesionales que son inabordables mediante las vías formales. De este modo, se infiere que todas las modalidades de formación cumplen un propósito diferencial dependiendo del contexto y no son sustituyentes, sino complementarias. En este marco, se presume que las Ecologías de Aprendizaje brindan un modelo holístico, complejo y profundo capaz de dar cabida a todos los elementos que forman parte del proceso de aprendizaje relativo al desarrollo profesional del docente (González-Sanmamed, SoutoSeijo, et al., 2019; Sangrà, Estévez, et al., 2019). Esto convierte a la metáfora ecológica en una opción adecuada para seguir interpretando y analizando los procesos de aprendizaje y actualización profesional del profesorado, respetando todas las formas en las que pueden ocurrir (Looi, 2001). Asimismo, el empleo de la tradición de investigación de Estudio de Caso se hace ineludible dado el carácter incipiente, complejo, multidimensional y poliédrico del constructo ecológico, inabordable por una aproximación cuantitativa (González-Sanmamed, Muñoz-Carril, \& Santos-Caamaño, 2019).

La principal limitación del estudio podría devenir por el número de participantes que componen el caso. Sería conveniente, de cara a futuras investigaciones, incrementar el número de informantes del caso para una descripción más profunda y holística de la realidad que rodea 
a los mecanismos y recursos de aprendizaje que los docentes ponen en marcha para aprender en los escenarios actuales.

Agradecimientos. Este estudio se ha elaborado en el marco del proyecto de investigación ECO4LEARN-HE, financiado por el Ministerio de Economía y Finanzas (Referencia EDU2015-67907R), y gracias a la financiación recibida por una de las autoras de esta investigación, Iris Estévez, en el programa FPI del Ministerio de Economía y Finanzas (BES-2016-077330).

\section{REFERENCIAS}

Castañeda, L. J., \& Adell, J. (2013). Entornos Personales de Aprendizaje: claves para el ecosistema educativo en red. Madrid, España: Editorial Marfil. Recuperado de https://cutt.ly/ersXzOn.

Cope, B., \& Kalantzis, M. (2009). Ubiquitous Learning. Illinois: University of Illinois Press. Recuperado de: https://bit.ly/2liWYU8 .

Creswell, J. W., \& Poth, C. N. (2018). Qualitative inquiry and research design: Choosing among five traditions. $\left(5^{\text {th }}\right.$ ed). London, UK: Sage Publications.

Denzin, N. K. (2015). Triangulation. The Blackwell Enyclopedia of Sociology. https://doi.org/10.1002/9781405165518.wbeost050.

González-Sanmamed, M., Estévez, I., Souto-Seijo, A., \& Muñoz-Carril, P.-C. (2020). Digital learning ecologies and professional development of university professors. Comunicar, 28(62), 9-18. doi: 10.3916/C62-2020-01

González-Sanmamed, M., Muñoz-Carril, P. C., \& Santos-Caamaño, F. J. (2019). Key components of learning ecologies: A Delphi assessment. British Journal of Educational Technology, 5(4), 1639-1655. doi: https://doi.org/10.1111/bjet.12805.

González-Sanmamed, M., Sangrà, A., Souto-Seijo, A., \& Estévez Blanco, I. (2018). Ecologías de aprendizaje en la Era Digital: desafíos para la Educación Superior. Publicaciones, 48(1), 25-45. doi: 10.30827/publicaciones.v48i1.7329.

González-Sanmamed, M., Souto-Seijo, A., González, I., \& Estévez, I. (2019). Aprendizaje informal y desarrollo profesional: análisis de las ecologías de aprendizaje del profesorado de educación infantil. Edutec. Revista Electrónica De Tecnología Educativa, (68), 70-81. doi: 10.21556/edutec.2019.68.1305.

Grané, M. (2009). Contextos, medios y herramientas 2.0 en la práctica educativa. En M. Grané, \& C. Willem (Coords.), Web 2.0: nuevas formas de aprender y participar (pp.131-157). Barcelona, España: Laertes educación.

Guglielmino, L. (2008). Why self-directed learning? International Journal of Self-Directed Learning, 5(1).

Huei-Tse Hou, Kuo-En Chang, \& Yao-Ting Sung (2009). Using blogs as a professional development tool for teachers: analysis of interaction behavioral patterns. Interactive Learning Environments, 17(4), 325-340, doi: 10.1080/10494820903195215.

Jackson, N. J. (2013). The concept of learning ecologies. Lifewide learning, education and personal development e-book. Recuperado de https://cutt.ly/LrsB29C.

Jorrín-Abellán, I. M. (2016). Hopscotch building: A model for the generation of qualitative research designs. Georgia Educational Researcher, 13(1). doi: 10.20429/ger.2016.130104.

Looi, C. K. (2001). Enhancing learning ecology on the Internet. Journal of Computer Assisted Learning, 17(1), 1320. doi: 10.1111/j.1365-2729.2001.00155.x. 
Macdonald, B., \& Walker, R. (1975). Case study and the Social Philosophy of education research. Cambridge Journal of Education, 5(1), 2-11. doi: 10.1080/0305764750050101.

Maina, M., \& Garcia, I. (2016). Articulating personal pedagogies through learning ecologies. En B. Gros, Kinshuk, \& M. Maina (Eds.), The future of Ubiquitous Learning: Learning designs for emerging pedagogies. Lecture notes in educational technology (pp. 73-94). Springer. doi: 10.1007/978-3-662-47724-3.

Marcelo, C. (2010). Autoformación para el siglo XXI. En J. Gairín (Coord.) Nuevas estrategias formativas para las organizaciones (pp. 141-170). Madrid, España: Wolters Kluwer.

Mayorga Fernández, M. J. (2004). 2004. Revista Electrónica de Investigación y Evaluación Educativa, 10(1), 2339. Recuperado de http://www.uv.es/RELIEVE/v10n1/RELIEVEv10n1_2.pdf .

Miles, M. B., Huberman, A. M., \& Saldaña, J. (2014). Qualitative data analysis: A methods sourcebook and the coding manual for qualitative researchers ( ${ }^{\text {rd }}$ ed). Thousands Oaks, CA, USA: Sage Publications. doi: 10.1080/10572252.2015.975966.

Munarriz, B. (1992). Técnicas y métodos en investigación cualitativa. En J. M. Muñoz Cantero, \& E. Abalde Paz (Coords.), Metodología educativa I (pp. 101- 116). A Coruña, España: Servicio de Publicaciones de la Universidad de A Coruña.

Patton, M. (2002). Qualitative research and evaluation methods ( $3^{a}$ ed). Thousand Oaks, CA, USA Sage Publications.

Sangrà, A., Estévez, I., Iglesias, V., \& Souto-Seijo, A. (2019). Desarrollo profesional docente a través de las ecologías de aprendizaje: Perspectivas del profesorado. Edutec. Revista Electrónica De Tecnología Educativa, (68), 42-53. doi: 10.21556/edutec.2019.68.1307.

Sangrà, A., González-Sanmamed, M., \& Guitert, M. (2013, October). Learning ecologies: Informal professional development opportunities for teachers. En 2013 IEEE 63rd Annual Conference International Council for Education Media (ICEM) (pp. 1-2). IEEE.

Stake, R. E. (2005). Qualitative case studies. En N. K. Denzin, \& Y. S. Lincoln (Eds.), The Sage handbook of qualitative research ( $3^{\text {rd }}$ ed) (pp. 443-466). Thousand Oaks, CA, USA: Sage.

Stake, R. E. (2010). Qualitative Research: Studying how things work. New York, NY, USA: The Guilford Press. 